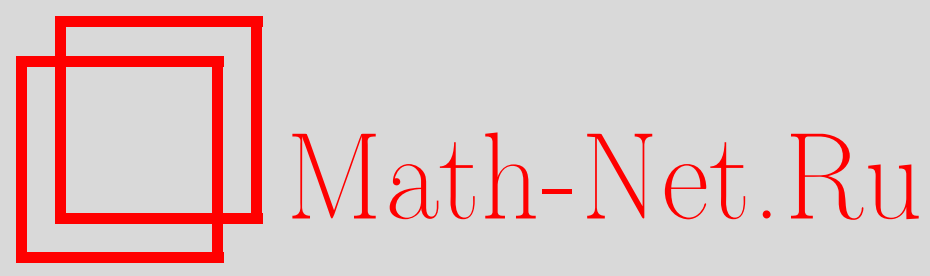

В. И. Юдович, О неограниченном росте вихря и циркуляции скорости течений стратифицированной и однородной жидкости, Матем. заметки, 2000, том 68, выпуск 4, 627636

DOI: https://doi.org/10.4213/mzm983

Использование Общероссийского математического портала Math-Net.Ru подразумевает, что вы прочитали и согласны с пользовательским соглашением http://www.mathnet.ru/rus/agreement

Параметры загрузки:

IP : 54.197 .217 .227

26 апреля 2023 г., 12:44:37

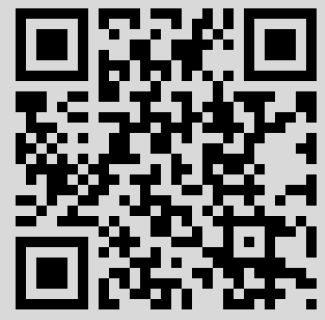




\title{
О НЕОГРАНИЧЕННОМ РОСТЕ ВИХРЯ И ЦИРКУЛЯЦИИ СКОРОСТИ ТЕЧЕНИЙ СТРАТИФИЦИРОВАННОЙ И ОДНОРОДНОЙ ЖИДКОСТИ
}

\section{В.И. Юдович}

\begin{abstract}
Рассмотрена система Обербека-Буссинеска без диссипации (идеальная конвекция) в горизонтальном слое или в "бочке" с плоскими дном и крышкой. Показано, что циркуляция скорости по жидкому контуру, состоящему из двух жидких кривых на дне и на крышке, соединенных парой изотермических жидких кривых, может быть вычислена явно и является линейной функцией времени. На стратифицированную жидкость распространен результат Серра о линейном росте азимутальной компоненты вихря вращательно симметричного течения идеальной жидкости между соосными цилиндрами. Доказано, что все плоские и осесимметричные изотермические течения в слое или в бочке неустойчивы относительно неизотермических возмущений, а в случае однородной жидкости все осесимметричные течения между соосными цилиндрами неустойчивы по Ляпунову относительно возмущения азимутальной компоненты скорости в метрике, включающей максимум модуля вихря.

Библиография: 7 названий.
\end{abstract}

Данная статья примькает к работе [1] (печатается в журнале Chaos).

Установлено, что при определенных условиях циркулящия скорости течения идеальной стратифицированной по температуре жидкости (уравнения Обербека-Буссинеска без вязкости и теплопроводности) может неограниченно (линейно) расти со временем. Это достигается непосредственньп вычислением циркуляции для специально выбранных контуров в случае течений в горизонтальном слое или в “бочке” с плоскими дном и крьшкой и ребром на их стыках с боковой поверхностью. Для плоских и осесимметричных течений из роста циркуляции следует неограниченньй рост вихря.

На стратифицированную жидкость распространен результат Серра [2] о линейном росте азимутальной компоненты вихря вращательно симметричного течения идеальной жидкости между соосными цилиндрами.

Конечно, действие вязкости и теплопроводности должно подавить неограниченньй рост, хотя это “физически очевидное” утверждение удается доказать лишь для двумерных течений, а в трехмерном случае известны лишь оценки вихря в интегральных нормах.

Работа выполнена при финансовой поддержке Российского фонда фундаментальных исследований, грант № 99-01-01023. 
Знание условий роста вихря помогает доказьвать неустойчивость течений самым прямым методом - предъявляя растущие возмущения. Так получается, что все плоские и осесимметричные изотермические течения в слое или в бочке неустойчивы относительно неизотермических возмущений, а в случае однородной жидкости все осесимметричные течения между соосньми цилиндрами неустойчивы относительно возмущения азимутальной компоненты скорости. Имеется в виду неустойчивость по Ляпунову в метрике, включающей максимум модуля вихря. Напомним, что выбор метрики играет решающую роль в вопросах устойчивости течений идеальной жидкости и иных сред без диссипации (см. [1], [3]).

Мы имеем здесь дело с уравнениями, для которых проблема глобальной разрешимости начально-краевых задач принципиально трудна и долгое время остается нерешенной. Несмотря на то, что вслед за Арнольдом [4] Должанский и Вишик [5] построили красивую аналитико-динамическую модель идеальной конвекции (бесконечномерное твердое тело в потенциальном поле сил), глобальную разрешимость не удается доказать даже для двумерных течений. Другая простейшая задача среди нерешенных относится к вращательно симметричным изотермическим течениям. В такой ситуации нам приходится, считая все задаваемые функции и поверхности $C^{\infty}$-гладкими, предполагать глобальное существование достаточно гладких решений. Единственность в этом случае очевидна. Замечу, что на заключения о неустойчивости сделанные предположения не влияют ведь случай появления сингулярности относится к неустойчивости.

Проблема возможности появления сингулярности (коллапса, взрыва) в течениях несжимаемой идеальной или вязкой жидкости в конечньй момент времени - возможно, самая волнующая в математической гидродинамике. Результаты работ [1]-[3], а также и данной статьи говорят о том, что возникновение сингулярности, выражающееся в потере гладкости поля скорости за бесконечное время ("медленньй взрьв"), вполне возможно.

1. Течения стратифицированной жидкости с растущей циркуляцией. Рассматривается идеальная конвекция жидкости в слое между твердыми горизонтальньми стенками $z=z_{0}$ и $z=z_{1}\left(x_{1}, x_{2}, x_{3}=z\right.$ - декартовы координаты). При отсутствии вязкости и теплопроводности уравнения Обербека-Буссинеска имеют вид

$$
\begin{aligned}
& \frac{d \boldsymbol{v}}{d t}=-\nabla p+\theta k, \quad \operatorname{div} \boldsymbol{v}=0 \\
& \frac{d \theta}{d t}=0, \quad \frac{d}{d t}=\frac{\partial}{\partial t}+\boldsymbol{v} \cdot \nabla
\end{aligned}
$$

Здесь $\boldsymbol{k}$ - единичный вектор оси $z$, направленньй вверх, $\boldsymbol{v}$ - поле скорости, $p$ и $\theta$ - давление и температура, отсчитьваемые от некоторых равновесных значений. Скорость $v$ должна удовлетворять краевому условию: $v_{3}=0$ при $z=z_{0}, z_{1}$.

Пусть $\gamma_{t}$ - гладкий жидкий контур, начальньй контур $\gamma_{0}$ задается параметрически: $a=a(\sigma)$, где параметр $\sigma$ есть "метка" частицы. Если $X=X(a, t)$ - движение произвольной жидкой частицы, определяемое как решение задачи Коши: $\dot{X}=\boldsymbol{v}(X, t)$, $X(a, 0)=a$, то уравнение контура $\gamma_{t}$ есть $x=x(\sigma, t)=X(a(\sigma), t)$.

Найдем скорость изменения циркулящии. Имеем

$$
\frac{d}{d t} \int_{\gamma_{t}} \boldsymbol{v} \cdot d \boldsymbol{x}=\frac{d}{d t} \int_{\gamma_{0}} \boldsymbol{v} \cdot \frac{\partial \boldsymbol{x}}{\partial \sigma} d \sigma=\int_{\gamma_{0}} \frac{d \boldsymbol{v}}{d t} \cdot \frac{\partial \boldsymbol{x}}{\partial \sigma} d \sigma+\int_{\gamma_{0}} \boldsymbol{v} \cdot \frac{\partial \boldsymbol{v}}{\partial \sigma} d \sigma
$$


Отсюда следует известная кинематическая формула

$$
\frac{d}{d t} \int_{\gamma_{t}} \boldsymbol{v} \cdot d \boldsymbol{x}=\int_{\gamma_{t}} \frac{d v}{d t} \cdot d \boldsymbol{x}
$$

Учет динамических уравнений (1) дает равенство

$$
\frac{d}{d t} \int_{\gamma_{t}} \boldsymbol{v} \cdot d \boldsymbol{x}=\int_{\gamma_{t}} \theta \boldsymbol{k} \cdot d \boldsymbol{x}=\int_{\gamma_{t}} \theta d z
$$

Отсюда видно, что при условии $\theta=$ const на $\gamma_{t}$ (это достаточно потребовать в начальньй момент правая часть равна нулю. Таким образом, ииркуляиия вдоль жидкого изотермического контура не меняется со временем.

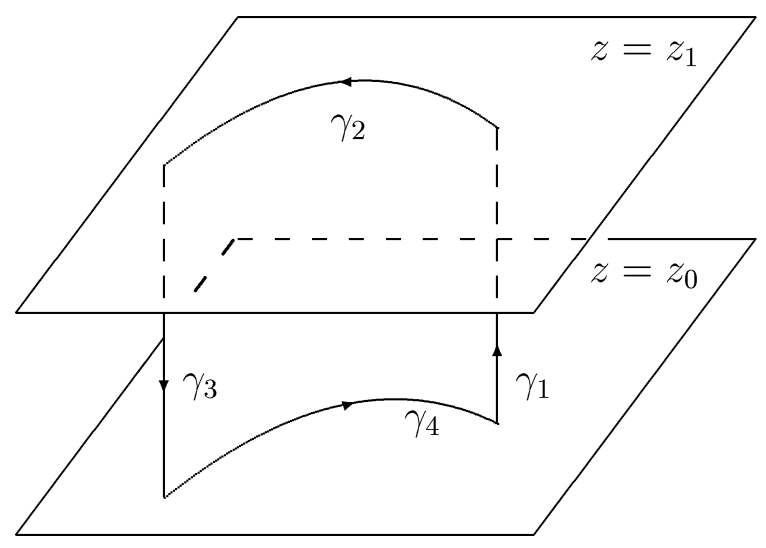

Рис. 1

Теперь рассмотрим контур $\gamma_{t}$, состоящий из четырех кусков (см. рис. 1). Предположим, что жидкие кривые $\gamma_{t}^{1}$ и $\gamma_{t}^{3}$ изотермичны, причем $\theta=\theta_{1}$ на $\gamma_{t}^{1}$ и $\theta=\theta_{3}$ на $\gamma_{t}^{3}$. Поскольку $d z=0$ на $\gamma_{t}^{2}$ и $\gamma_{t}^{4}$, равенство (3) принимает вид

$$
\frac{d}{d t} \int_{\gamma_{t}} \boldsymbol{v} \cdot d \boldsymbol{x}=\int_{\gamma_{t}^{1}} \theta d z+\int_{\gamma_{t}^{3}} \theta d z
$$

Интегралы вычисляются, и мы приходим к равенству

$$
\frac{d}{d t} \int_{\gamma_{t}} \boldsymbol{v} \cdot d \boldsymbol{x}=\left(\theta_{1}-\theta_{3}\right) h
$$

где $h=z_{1}-z_{0}$ - толщина слоя. Итак, при $\theta_{1} \neq \theta_{3}$ ииркуляиия скорости линейно растет со временем:

$$
\int_{\gamma_{t}} \boldsymbol{v} \cdot d \boldsymbol{x}=\int_{\gamma_{0}} \boldsymbol{v}_{0} \cdot d \boldsymbol{x}_{0}+t\left(\theta_{1}-\theta_{3}\right) h, \quad \boldsymbol{v}_{0}=\left.\boldsymbol{v}\right|_{t=0}
$$


Интересно получить асимптотическую форму равенств $(5),(6)$, когда контур $\gamma_{t}$ стягивается к кривой $\gamma_{t}^{1}$ (и $\theta_{3} \rightarrow \theta_{1}$ ). Пусть $S_{t}$ - некоторая кусочно-гладкая жидкая поверхность, натянутая на контур $\gamma_{t}$. По теореме Стокса циркуляция поля $\boldsymbol{v}$ вдоль $\gamma_{t}$ равна потоку вихря через $S_{t}$ :

$$
\int_{\gamma_{t}} \boldsymbol{v} \cdot d \boldsymbol{x}=\int_{S_{t}}(\operatorname{rot} \boldsymbol{v})_{n} d S .
$$

Предположим, что контур $\gamma_{t}^{1}$ регулярен, так что всюду на нем $\nabla \theta \neq 0$. Тогда локально определена поверхность уровня $\theta=\theta_{1}$, на которой лежит контур $\gamma_{t}^{1}$. Контур $\gamma_{t}^{3}$ тоже регулярен, когда он расположен достаточно близко к $\gamma_{t}^{1}$. Заметим, что регулярность жидкого контура сохраняется, если она имеет место в начальньй момент. Действительно, дифференцирование уравнения (2) дает для функций $\partial \theta / \partial x_{i}$ на движении жидкой частицы систему линейных обыкновенных дифференциальных уравнений с непрерывными коэффициентами

$$
\frac{d}{d t} \frac{\partial \theta}{\partial x_{i}}=-\frac{\partial v_{k}}{\partial x_{i}} \frac{\partial \theta}{\partial x_{k}}, \quad i, k=1,2,3,
$$

а ее решение не может обрашаться в нуль ни в какой момент $t$, если оно отлично от нуля в начальньй момент.

Пусть $s$ - длина дуги на $\gamma_{t}^{1}$, а $s \mapsto \ell(s)=\ell$ - поле единичных векторов на $\gamma_{t}^{1}$, касательных к $S_{t}$ и нормальных к $\gamma_{t}^{1}$. Тогда $\theta_{3}=\theta_{1}+\nabla \theta \cdot \ell d \ell$ с точностью до малых высшего порядка относительно $d \ell$ - "расстояния" между кривыми $\gamma_{t}^{1}$ и $\gamma_{t}^{3}$ в направлении $\ell\left(d \ell>0\right.$ при $\theta_{3}>\theta_{1}$ и $d \ell<0$, если $\left.\theta_{3}<\theta_{1}\right)$. Для $d \ell$ получается выражение

$$
d \ell=\frac{\theta_{3}-\theta_{1}}{\left|\nabla_{0} \theta^{0}\right|}
$$

где $\theta^{0}$ - сужение функции $\theta$ на $S_{t}$, a $\nabla_{0}$ - градиент вдоль поверхности $S_{t}$.

Далее, для бесконечно малых $\theta_{3}-\theta_{1}$ можно написать

$$
d S=d s d \ell=\frac{d s}{\left|\nabla_{0} \theta^{0}\right|}\left(\theta_{3}-\theta_{1}\right)
$$

Остается заметить, что $(\operatorname{rot} v)_{n}$ стремится к компоненте вихря вдоль нормали к $\gamma_{t}^{1}$, лежащей на поверхности уровня $\theta=\theta_{1}$. Таким образом, в пределе имеем

$$
(\operatorname{rot} \boldsymbol{v})_{n}=\frac{\operatorname{rot} \boldsymbol{v} \cdot(\boldsymbol{\tau} \wedge \nabla \theta)}{|\nabla \theta|}
$$

С использованием (7)-(9) из (5) получаем равенство

$$
\frac{d}{d t} \int_{\gamma_{t}^{1}} \frac{\nabla \theta \wedge \operatorname{rot} \boldsymbol{v}}{|\nabla \theta|^{2}} \cdot d \boldsymbol{x}=h, \quad d \boldsymbol{x}=\boldsymbol{\tau} d s .
$$

Мы заменили $\left|\nabla_{0} \theta^{0}\right|$ на $|\nabla \theta|$, так как компонента вектора $\nabla \theta$ вдоль поверхности $\theta=\theta_{1}$ равна нулю. Итак, интеграл в (10) по всякой изотермической и регулярной $(\nabla \theta \neq 0)$ кривой, соединяющей плоскости $z=z_{0} u z=z_{1}$, линейно растет со временем. 
Не обязательно рассматривать бесконечньй слой - предыдущие выводы сохраняют силу и в тех случаях, когда жидкость находится в цилиндре (с произвольным сечением) или даже в "бочке" - области, ограниченной двумя плоскими площадками: $\Sigma_{0}$ на плоскости $z=z_{0}, \Sigma_{1}$ на плоскости $z=z_{1},-$ и гладкой боковой поверхностью. Существенно, что на стьках боковой поверхности и граничных кривых $\partial \Sigma_{0}$ и $\partial \Sigma_{1}$ гладкость границы области течения теряется, образуются ребра. Это условие гарантирует невозможность ухода жидких частиц с дна $\Sigma_{0}$ и с крышки $\Sigma_{1}$ по теореме единственности для гладкого поля скорости $\boldsymbol{v}(x, t)$ (жидкие частицы на ребрах границы должны покоиться). В итоге жидкий контур $\gamma_{t}$ вечно сохраняет нужные нам свойства.

В общем трехмерном случае пока нет ответа на интригующий вопрос, что является причиной роста циркуляции или криволинейного интеграла (10). Таковыми могли бы быть рост вихря или рост длины контура $\gamma_{t}$. Последняя возможность особенно пикантна в случае, когда контур $\gamma_{t}$ остается в ограниченной области пространства (скажем, для течений в бочках). Что происходит - контур сильно извивается в области течения или сильно моршится? Неизвестно также, может ли $|\nabla \theta|$ затухать при $t \rightarrow \infty$ для жидкой частицы, что могло бы привести к росту интеграла (10). Хотя и трудно, но сейчас уже возможно ответить на эти вопросы прямыми вычислениями. Рискну предположить, что все перечисленные возможности могут реализоваться в различных течениях или даже для различных контуров в одном и том же течении. Кажется вероятным, что может встретиться и более быстрый рост циркуляции, чем линейный.

В плоском случае ситуация проще. Считая теперь, что вертикально вверх направлена ось $x_{2}$, имеем равенство

$$
\int_{\gamma_{t}} \boldsymbol{v} \cdot d \boldsymbol{x}=\int_{\Omega_{t}} \omega d x_{1} d x_{2},
$$

где $\omega=\omega_{3}\left(x_{1}, x_{2}, t\right)$ - единственная ненулевая компонента вихря, а $\Omega_{t}$ - площадка, ограниченная контуром $\gamma_{t}=\partial \Omega_{t}$. Площадь $\left|\Omega_{t}\right|$ жидкой области $\Omega_{t}$ не меняется со временем: $\left|\Omega_{t}\right|=\left|\Omega_{0}\right|$, и интеграл (11) по модулю не превосходит $\|\omega(\cdot, t)\|_{L_{\infty}\left(\Omega_{t}\right)} \cdot\left|\Omega_{0}\right|$. Поэтому из (4) следует, что существование в начальный момент невырожденной линии уровня $\theta=\theta_{1}$ (на ней всюду $|\nabla \theta| \neq 0$ ) влечет неограниченный рост максимума модуля вихря по области течения. Этот вьвод, конечно, распространяется и на осесимметричные (с нулевой азимутальной компонентой) течения. Хотя и природа не изобилует причинами, может быть росту циркуляции способствует неограниченньй рост длины жидкого контура.

Согласно результатам Арнольда [4] существует много устойчивых плоских и осесимметричных изотермических течений жидкости. Однако, всякое плоское или осесимметричное (ось вертикальна) изотермическое $(\theta=$ const всюду и всегда) стационарное решение системы (1), (2) в горизонтальном слое неустойчиво относительно неизотермических возмущений в метрике, включающей $\|\operatorname{rot} v\|_{L_{\infty}}$. Для доказательства достаточно в начальньй момент $t=0$ организовать возмущение температуры, допускающее существование такого контура, как на рис. 1. Это возмущение можно взять $C^{\infty}$-гладким, сколь угодно мальм в метрике $C^{k}$ ( $k$ любое) и локальным -c носителем в малой окрестности какой-нибудь вертикальной прямой. Например, достаточно положить $\theta(x)=x_{1} \chi(x)$, где $\chi$ - соответствующая срезающая функция.

\section{2. Рост азимутальной компоненты вихря во вращательно симметричных} течениях. Теперь рассмотрим врашательно симметричные решения системы $(1),(2)$. 
Для компонент скорости $u, v, w$ в цилиндрических координатах $r, \varphi, z$ (ось $z$ направлена вертикально вверх), температуры $\theta$ и давления $p$ в случае, когда все эти функции не зависят от $\varphi$, имеем систему

$$
\begin{aligned}
& \frac{d u}{d t}-\frac{v^{2}}{r}=-p_{r}, \quad \frac{d w}{d t}=-p_{z}+\theta, \quad u_{r}+\frac{u}{r}+w_{z}=0 \\
& \frac{d q}{d t}=0, \quad \frac{d \theta}{d t}=0, \quad q=r v, \quad \frac{d}{d t}=\frac{\partial}{\partial t}+u \partial_{r}+w \partial_{z}
\end{aligned}
$$

Будем считать, что жидкость располагается в зазоре между цилиндрами $r=r_{1}$ и $r=r_{2}>r_{1}$. Из дальнейшего будет видно, что результаты без изменения переносятся и на кольцевой цилиндр конечной длины ("шайбу") или даже на аналогичные области с кривыми дном и крышкой, которые однако должны быть вращательно симметричными гладкими поверхностями, трансверсальными к цилиндрам.

Обозначая через $\omega^{r}, \omega^{\varphi}, \omega^{z}$ цилиндрические компоненты вихря rot $v$, запишем уравнения Гельмгольца в виде

$$
\begin{aligned}
\frac{d \omega^{r}}{d t} & =\omega^{r} u_{r}+\omega^{z} u_{z} \\
\frac{d \omega^{\varphi}}{d t} & =\frac{2 v v_{z}}{r}+\frac{\omega^{\varphi} u}{r}-\theta_{r} \\
\frac{d \omega^{z}}{d t} & =\omega^{r} w_{r}+\omega^{z} w_{z} .
\end{aligned}
$$

При этом $\omega^{r}=-v_{z}=-q_{z} / r, \omega^{\varphi}=u_{z}-w_{r}, \omega^{z}=v_{r}+v / r=q_{r} / r$. Второе уравнение системы (14) для $\omega^{\varphi}$ можно записать в виде

$$
\frac{d}{d t} \frac{\omega^{\varphi}}{r}=\frac{\partial}{\partial z} \frac{q^{2}}{r^{4}}-\frac{1}{r} \theta_{r} .
$$

Предположим, что существует пара жидких поверхностей вращения $S_{t}^{1}$ и $S_{t}^{2}$, которые трансверсально пересекают оба цилиндра при всех $t$, являются поверхностями уровня функции $q=r v$ (удельного момента количества движения), так что $q=q_{1}$ на $S_{t}^{1}$ и $q=q_{2}$ на $S_{t}^{2}$, и вместе с цилиндрами ограничивают жидкую область $D_{t}$ (см. рис. 2 ). Достаточно задать такие поверхности $S_{0}^{1}$ и $S_{0}^{2}$ при $t=0$. Тогда, поскольку величина $q$ сохраняется для каждой жидкой частицы, жидкие поверхности $S_{t}^{1}$ и $S_{t}^{2}$ определяются для любого $t$ - в предположении, что гладкое решение системы $(12),(13)$ существует. В силу единственности решения задачи Коши для уравнений движения жидких частиц

$$
\begin{gathered}
\dot{r}=u, \quad r \dot{\varphi}=v, \quad \dot{z}=w, \\
\left.r\right|_{t=0}=r_{0},\left.\quad \varphi\right|_{t=0}=\varphi_{0},\left.\quad z\right|_{t=0}=z_{0},
\end{gathered}
$$

поверхности $S_{t}^{1}$ и $S_{t}^{2}$ не могут пересекаться и на них не могут образовьваться самопересечения. Из рассмотрения линейной системы для производных $q_{r}, q_{z}$ вдоль движения жидкой частицы нетрудно вьвести, что поверхности $S_{t}^{1}$ и $S_{t}^{2}$ вечно остаются регулярными ( $\nabla q \neq 0$ на них всюду), если они таковы в начальньй момент. 


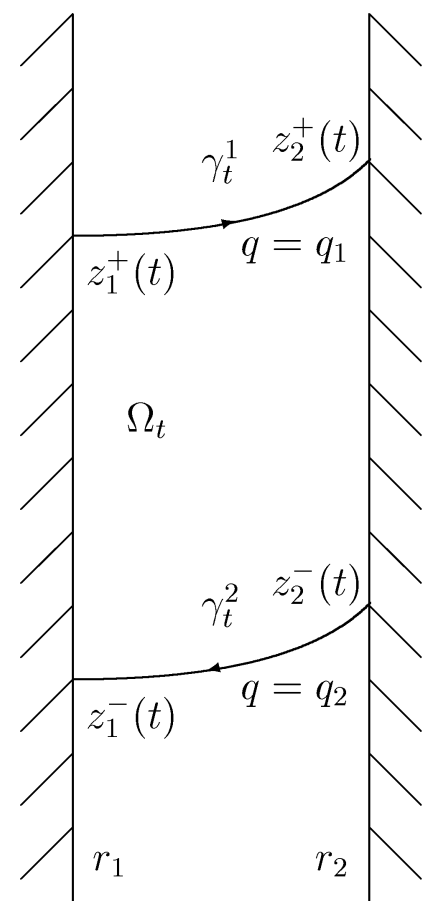

Рис. 2

Применяя формулу дифференцирования интеграла по области, зависящей от времени, с учетом несжимаемости жидкости получаем

$$
\frac{d}{d t} \int_{D_{t}} \frac{\omega^{\varphi}}{r} d x=\int_{D_{t}} \frac{d}{d t} \frac{\omega^{\varphi}}{r} d x, \quad d x=r d r d \varphi d z
$$

Используя уравнение (14) и интегрируя по частям, вьводим

$$
\frac{d}{d t} \int_{D_{t}} \frac{\omega^{\varphi}}{r} d x=\int_{\partial D_{t}} \frac{q^{2}}{r^{4}} n_{z} d S-2 \pi \int_{z_{2}^{-}}^{z_{2}^{+}} \theta\left(r_{2}, z, t\right) d z+2 \pi \int_{z_{1}^{-}}^{z_{1}^{+}} \theta\left(r_{1}, z, t\right) d z .
$$

Здесь $z_{1}^{+}, z_{2}^{+}$- аппликаты верхних, а $z_{1}^{-}, z_{2}^{-}$- нижних концов тех частей контура $\gamma_{t}$, которые лежат на цилиндрах $r=r_{1}$ и $r=r_{2}$. Далее, $n_{z}$ есть $z$-компонента орта внешней нормали $\boldsymbol{n}$. Поскольку на цилиндрах $n_{z}=0$, интеграл по $\partial D_{t}$ сводится к сумме интегралов по $S_{t}^{1}$ и $S_{t}^{2}$.

Пусть $\gamma_{t}^{1}, \gamma_{t}^{2}$ и $\Omega_{t}$ - меридиональные сечения соответственно поверхностей $S_{t}^{1}, S_{t}^{2}$ и области $D_{t}$ (см. рис. 2). После интегрирования по углу $\varphi$ равенство (16) принимает вид

$$
\frac{d}{d t} \int_{\Omega_{t}} \omega^{\varphi} d r d z=q_{1}^{2} \int_{\gamma_{t}^{1}} \frac{n_{z}}{r^{3}} d s+q_{2}^{2} \int_{\gamma_{t}^{2}} \frac{n_{z}}{r^{3}} d s-\int_{z_{2}^{-}}^{z_{2}^{+}} \theta\left(r_{2}, z, t\right) d z+\int_{z_{1}^{-}}^{z_{1}^{+}} \theta\left(r_{1}, z, t\right) d z .
$$

Интегралы по $\gamma_{t}^{1}$ и $\gamma_{t}^{2}$ вычисляются явно. Если ориентированная кривая $\gamma_{t}^{1}$ задана натуральными уравнениями $r=r_{0}(s), z=z_{0}(s)$ ( $s$ - длина дуги), то касательный орт $\boldsymbol{\tau}$ 
и орт $\boldsymbol{n}$ внешней к $\Omega_{t}$ нормали суть $\boldsymbol{\tau}=\left(r_{0}^{\prime}(s), z_{0}^{\prime}(s)\right)$ и $\boldsymbol{n}=\left(-z_{0}^{\prime}(s), r_{0}^{\prime}(s)\right)$. Таким образом, $n_{z}=r_{0}^{\prime}(s)$ и вьполняется соотношение

$$
\int_{\gamma_{t}^{1}} \frac{n_{z}}{r^{3}} d s=\int_{\gamma_{t}^{1}} \frac{r_{0}^{\prime}(s) d s}{r_{0}^{3}(s)}=\int_{\gamma_{t}^{1}} \frac{d r}{r^{3}}=\frac{1}{2}\left(\frac{1}{r_{1}^{2}}-\frac{1}{r_{2}^{2}}\right)
$$

Интеграл по $\gamma_{t}^{2}$ вычисляется аналогично. В результате из (17) получаем равенство

$$
\frac{d}{d t} \int_{\Omega_{t}} \omega^{\varphi} d r d z=\frac{1}{2}\left(q_{1}^{2}-q_{2}^{2}\right)\left(\frac{1}{r_{1}^{2}}-\frac{1}{r_{2}^{2}}\right)-\int_{z_{2}^{-}}^{z_{2}^{+}} \theta\left(r_{2}, z, t\right) d z+\int_{z_{1}^{-}}^{z_{1}^{+}} \theta\left(r_{1}, z, t\right) d z
$$

Эта красивая выкладка в изотермическом случае была проведена Серром [2]. Рассуждая, как при выводе формулы (10), приходим к следующей формуле, получаемой в результате стягивания сечения $\Omega_{t}$ к кривой $\gamma_{t}^{1}$ :

$$
\frac{d}{d t} \int_{\Omega_{t}} \frac{\omega^{\varphi}}{|\nabla q|} d s=q_{1}\left(\frac{1}{r_{1}^{2}}-\frac{1}{r_{2}^{2}}\right)-\frac{\theta\left(r_{2}, z_{2}^{+}(t), t\right)}{q_{z}\left(r_{2}, z_{2}^{+}(t), t\right)}+\frac{\theta\left(r_{1}, z_{1}^{+}(t), t\right)}{q_{z}\left(r_{1}, z_{1}^{+}(t), t\right)}
$$

Что касается знаменателей в последних членах равенства (19), достаточно потребовать, чтобы они были отличны от нуля в начальный момент. Действительно, $u=0$ при $r=r_{1}, r_{2}$. Поэтому $\theta_{z}$ удовлетворяет на цилиндрах уравнению

$$
\frac{d \theta_{z}}{d t}=-w_{r} \theta_{z}
$$

из которого следует, что для каждой частицы $\theta_{z}$ не меняет знака и не может от ненулевого значения прийти к нулевому.

При постоянном $\theta$ и $\left|q_{1}\right| \neq\left|q_{2}\right|$ получается, что $\int_{\Omega_{t}} \omega^{\varphi} d r d z$ есть линейная функция времени, откуда следует, что $\max \left|\omega^{\varphi} / r\right|$ по $\Omega_{t}$ линейно растет при $t \rightarrow \pm \infty$ [2]. Формула (18) указьвает на второй механизм роста $\omega^{\varphi}$, связанньй со стратификацией. Он работает, когда температура $\theta$ на каждом из цилиндров сохраняет знак, причем знаки при $r=r_{1}$ и $r=r_{2}$ противоположны; достаточно этого потребовать от начальной температуры $\theta^{0}$.

Два указанных механизма могут конкурировать или работать кооперативно. В случае конкуренции результат неясен - впрочем, конечно, вывод о линейном росте сохраняется, когда температура $\theta$ при $r=r_{1}, r_{2}$ достаточно мала (в начальный момент, а тогда и вечно).

Когда действия вращения и стратификации направлены в одну сторону, рост величины $\max _{\Omega_{t}}\left|\omega^{\varphi} / r\right|$ остается не менее чем линейньм. Пусть, например, $q_{1}^{2}>q_{2}^{2} u \partial$ ля $^{2}$ начальной температуры при любом z выполняются неравенства

$$
\theta\left(r_{1}, z, 0\right) \geqslant 0, \quad \theta\left(r_{2}, z, 0\right) \leqslant 0
$$

Тогда из (18) следует оченка снизу $\left(\omega_{0}^{\varphi}=\left.\omega^{\varphi}\right|_{t=0}\right)$

$$
\int_{\Omega_{t}} \omega^{\varphi} d r d z \geqslant \int_{\Omega_{t}} \omega_{0}^{\varphi} d r d z+\frac{1}{2} t\left(q_{1}^{2}-q_{2}^{2}\right)\left(\frac{1}{r_{1}^{2}}-\frac{1}{r_{2}^{2}}\right)
$$


Ввиду несжимаемости жидкости

$$
\int_{\Omega_{t}} r d r d z=\frac{\left|D_{t}\right|}{2 \pi}
$$

не зависит от $t$, так что из (20) следует, что

$$
\max _{\Omega_{t}}\left|\frac{\omega^{\varphi}}{r}\right| \rightarrow \infty \quad \text { при } t \rightarrow+\infty
$$

(аналогично устанавливается, что то же верно и для $t \rightarrow-\infty$ ).

3. Потеря гладкости вращательно симметричных течений и неустойчивость осесимметричных течений. Сейчас речь пойдет об изотермических вращательно симметричных течениях - в (12), (13) $\theta \equiv 0$. Сначала мы опишем класс течений, для которых растут со временем компоненты вихря $\omega^{r}, \omega^{z}$ (а $\omega^{\varphi}$ не растет). Из уравнений (12), (13) следует равенство

$$
\frac{d}{d t} \frac{q_{r} q_{z}}{r}=-\frac{w_{r}}{r} q_{z}^{2}-\frac{u_{z}}{r} q_{r}^{2} .
$$

Если жидкая частица двигается вдоль твердой поверхности $z=z_{0}$, то на всей ее траектории $w=0, w_{r}=0, \omega^{\varphi}=u_{z}$. Тогда (21) принимает вид

$$
\frac{d}{d t} \frac{q_{r} q_{z}}{r}=-\frac{\omega^{\varphi}}{r} q_{r}^{2} .
$$

Предположим еще, что для данной частицы не только $\theta$, но и $q=0$. Тогда согласно $(15)$

$$
\frac{d}{d t} \frac{\omega^{\varphi}}{r}=0
$$

Из (22), (23) нетрудно вывести (см. [1, лемма 5.1]), что если для данной частицы вьполнено $\omega^{\varphi} q_{r} q_{z}<0$, то $q_{r}^{2}+q_{z}^{2} \rightarrow \infty$ или, что все равно, $\left(\omega^{r}\right)^{2}+\left(\omega^{z}\right)^{2} \rightarrow \infty$ с вероятностью 1. Это означает, что

$$
\frac{1}{T} \operatorname{mes}\left\{t: 0 \leqslant t \leqslant T, q_{r}^{2}+q_{z}^{2}>N^{2}\right\} \rightarrow 1
$$

каково бы ни было $N$.

Если же частица двигается вдоль непроницаемого цилиндра $r=r_{0}$, то на ее траектории $u=0, u_{z}=0, \omega^{\varphi}=-w_{r}$,

$$
\frac{d}{d t} \frac{q_{r} q_{z}}{r}=\frac{\omega^{\varphi}}{r} q_{z}^{2} .
$$

Снова предполагая, что на этой траектории $q=0$, можно заключить, что $q_{r}^{2}+q_{z}^{2} \rightarrow \infty$ при $t \rightarrow \infty$ с вероятностью 1 , если в начальный момент для данной жидкой частицы $\omega^{\varphi} q_{r} q_{z}>0$. Повторяя рассуждения из $\S 5$ работы [1], можно прийти к выводу, что все стационарные вращательно симметричные движения, обладающие хотя бы одной траекторией, лежсащей на твердой плоскости $z=z_{0}$ или на твердом чилиндре $r=r_{0}$, причем на этой траектории $q=0$, неустойчивы в метрике вихря. 
Существование стационарных течений с линией тока, обладающей требуемыми свойствами, нетрудно установить, пользуясь общим представлением стационарного вращательно симметричного течения [6], [7].

Плоские стационарные движения неустойчивы относительно трехмерных возмущений в метрике вихря [1]. Можно предположить, что стационарные осесимметричные течения неустойчивы относительно вращательно симметричных возмущений. В общем случае это не доказано. Однако, произвольное (дажсе не обязательно стационарное) осесимметричное течение между соосными иилиндрами неустойчиво относительно малых вращательно симметричных возмущений в метрике вихря $(\max |\operatorname{rot} \boldsymbol{v}(\cdot, t)|+\boldsymbol{м} \Omega$. норма). Для доказательства достаточно в начальньй момент, не трогая компоненты $u$ и $w$, задать компоненту $v$ так, чтобы существовал такой контур, как на рис. 2. При этом $v$ можно выбрать $C^{\infty}$-гладким, сколь угодно мальм в метрике $C^{k}$ и с носителем в малой окрестности какого нибудь отрезка $z=z_{0}$. Неустойчивость сразу следует из (17). Можно предположить, что всякое осесимметричное течение неустойчиво относительно вращательно симметричных возмущений [1]. Общий результат неизвестен.

\section{СПИСОК ЦИТИРОВАННОЙ ЛИТЕРАТУРЫ}

[1] Юдович В.И.О порче гладкости решений уравнений Эйлера и неустойчивости, присущей течениям идеальной жидкости // Деп. ВИНИТИ 22.07.98, № 2331-В98. М.: ВИНИТИ, 1998.

[2] Serre D. La croissance de la vorticité dans les écoulements parfaits incompressibles // Comp. Rend. Acad. Sci. Paris Sér. I. 1999. V. 328. P. 549-552.

[3] Юдович В. И. О потере гладкости решений уравнений Эйлера со временем // Сб. “Динамика сплошной среды". Вып. 16. Новосибирск, 1974. С. 71-78.

[4] Arnold V. I. Sur la géometrie differéntielle des groupes de Lie de dimension infinie et ses applications á l’hydrodynamique des fluides parfaits // Ann. Inst. Fourier. 1966. V. 16. № 1. Р. 316-361.

[5] Гледзер Е. Б., Должанский Ф. В., Обухов А. М. Системы гидродинамического типа и их применение. М.: Наука, 1981.

[6] Васильев О.Ф. Основы механики винтовых и циркуляционных потоков. Ленинград: Госэнергоиздат, 1958.

[7] Meissel E. Über den Ausfluss des Wassers aus Gefässen in zwei besonderen Fällen nach Eintritt des Beharrungszustandes // Archiv der Mathematik und Physik. 1873. V. 55. 